\title{
High Velocity Cloud Complex H: A Satellite of the Milky Way in a Retrograde Orbit?
}

\author{
Felix J. Lockman \\ National Radio Astronomy Observatory 1, P.O. Box 2, Green Bank, WV, 24944; \\ jlockman@nrao.edu
}

\begin{abstract}
Observations with the Green Bank Telescope of $21 \mathrm{~cm} \mathrm{H}$ I emission from the highvelocity cloud Complex $\mathrm{H}$ suggest that it is interacting with the Milky Way. A model in which the cloud is a satellite of the Galaxy in an inclined, retrograde circular orbit reproduces both the cloud's average velocity and its velocity gradient with latitude. The model places Complex $\mathrm{H}$ at $R=33 \pm 9 \mathrm{kpc}$ from the Galactic Center on a retrograde orbit inclined $\approx 45^{\circ}$ to the Galactic plane. At this location it has a mass in $\mathrm{H}$ I of $>6 \times 10^{6} M_{\odot}$ and dimensions of at least $10 \times 5 \mathrm{kpc}$. Some of the diffuse H I associated with the cloud has apparently been decelerated by interaction with Galactic gas. Complex $\mathrm{H}$ has similarities to the dwarf irregular galaxy Leo $\mathrm{A}$ and to some compact high-velocity clouds, and has an internal structure nearly identical to parts of the Magellanic Stream, with a pressure $P / k \approx 100 \mathrm{~cm}^{-3} \mathrm{~K}$.
\end{abstract}

Subject headings: Galaxy:halo — Galaxy: structure — ISM:clouds - Radio Lines:ISM

\section{Introduction}

The current model for galaxy formation is one of hierarchical aggregation (Freeman \& BlandHawthorn 2002), and in this context the problem of the nature of high-velocity H I clouds has attracted renewed attention (Blitz et al. 1999). The high-velocity H I cloud "Complex H", first discovered by Dieter (1971) and Hulsbosch (1971) and shown in Figure 1, covers more than 100 square degrees at very low Galactic latitude. Its brighter components have a velocity with respect to the Local Standard of Rest of $V_{L S R} \approx-200 \mathrm{~km} \mathrm{~s}^{-1}$, which, in this direction, is at least $35 \mathrm{~km}$ $\mathrm{s}^{-1}$ more negative than is allowed for an object in circular Galactic rotation at any distance, and about $100 \mathrm{~km} \mathrm{~s}^{-1}$ more negative than the observed minimum velocity from $\mathrm{H}$ i in the Galactic disk. It lies in the Galactic plane, so the anomalous motion cannot be attributed to infall.

\footnotetext{
${ }^{1}$ The National Radio Astronomy Observatory is a facility of the National Science Foundation operated under cooperative agreement with Associated Universities, Inc.
} 
Wakker et al. (1998) show that Complex H must be more than $5 \mathrm{kpc}$ from the Sun, which eliminates a possible association with the Local Bubble, and they dismiss several other "local" models on the grounds that the cloud does not seem to show strong interaction with Galactic gas. Morras, Bajaja, \& Arnal (1998), however, note that Complex H overlaps in position with a large $\mathrm{H}$ I shell at permitted velocities, and propose that it created the shell as it penetrated the Galactic disk about $22 \mathrm{kpc}$ from the Galactic Center. This interpretation has been challenged by Blitz et al. (1999), who cite a lack of ancillary evidence for so energetic an encounter and, instead, hold Complex $\mathrm{H}$ to be an important link in a chain of evidence which leads them to conclude that most high-velocity clouds are at intergalactic rather than interstellar distances.

In fact, the basic conclusion that the velocity of Complex $\mathrm{H}$ cannot arise from circular rotation about the Galaxy is correct only for prograde orbits. An LSR velocity of $-200 \mathrm{~km} \mathrm{~s}^{-1}$ at $\ell=130^{\circ}$ can be achieved by an object with $V_{\theta} / R \approx-5 \mathrm{~km} \mathrm{~s}^{-1} \mathrm{kpc}^{-1}$, where $\mathrm{R}$ is the distance from the Galactic center, $V_{\theta}$ is the azimuthal velocity, and the negative sign indicates that the motion is against the sense of normal Galactic rotation. This Letter presents $21 \mathrm{~cm} \mathrm{H}$ I observations of Complex H made with the Green Bank Telescope which show that there is $\mathrm{H}$ I at velocities between the cloud and the disk, confirming previous suspicions that it is interacting with the Milky Way. The new data are consistent with a model in which this object is a satellite of the Milky Way in an inclined, retrograde orbit, whose outermost layers are currently being stripped away in its encounter with the Galaxy.

\section{Complex $\mathbf{H}$ and the Galactic Disk}

Figure 2 shows a cut through Complex $\mathrm{H}$ at $\ell=130.5$ made from the Leiden-Dwingeloo (LD) $21 \mathrm{~cm}$ H I survey data (Hartmann \& Burton 1997). The cloud divides into two distinct sections: a bright "core" which lies at $b \lesssim 5^{\circ}$, and a fainter, more diffuse "tail" at $b>5^{\circ}$. Both the core and the tail move to more positive velocities away from the cloud apex near $b \approx 4^{\circ}$, the tail reaching velocities allowed by Galactic rotation at $b \approx 10^{\circ}$. This same general structure is seen at many longitudes and was apparent in the earliest observations (Dieter 1971; Hulsbosch 1975). There is also a hint of faint $\mathrm{H}$ I emission at velocities between that of the cloud core and the Galactic disk.

Part of Complex H at $130^{\circ} .5 \leq \ell \leq 132.5$ and $-2^{\circ}<b<+8^{\circ}$ was observed in the $21 \mathrm{~cm}$ line with the Robert C. Byrd Green Bank Telescope (GBT) in August 2002 at $9^{\prime}$ angular resolution with $3^{\prime}$ spacing between spectra. Each spectrum covers $-300 \leq V_{L S R} \leq+200 \mathrm{~km} \mathrm{~s}^{-1}$ at $1.25 \mathrm{~km}$ $\mathrm{s}^{-1}$ velocity resolution. The $1 \sigma$ noise in a channel is $0.16 \mathrm{~K}$. The equipment and observing methods are identical to those described elsewhere (Lockman 2002).

Figure 3 shows the GBT data at $\ell=130.6$. About $40 \%$ of the spectra through the core of the Complex have two line components, one broad and one narrow, with a median $\Delta v$ (FWHM) of 8 and $28 \mathrm{~km} \mathrm{~s}^{-1}$. In contrast, lines from the tail are almost uniformly broad with a median $\Delta v=34$

$\mathrm{km} \mathrm{s}^{-1}$. The median width of all lines from the core is $22 \mathrm{~km} \mathrm{~s}^{-1}$, while almost every spectrum 
through the tail has $\Delta v>22 \mathrm{~km} \mathrm{~s}^{-1}$. This suggests that the division between core and tail is not arbitrary, but is related to the nature of the Complex. Fig. 3 also shows diffuse H I emission filling much of the gap in velocity between the Complex and the Galactic disk, strong evidence for an interaction between the Complex and the Milky Way. The Complex is not nearly as isolated from the Galaxy as it seemed from earlier observations.

\section{A Model for Complex $\mathbf{H}$}

In the model proposed here Complex $\mathrm{H}$ has a core which moves like a solid body on a circular orbit, and a tail of diffuse, decelerated gas resulting from its interaction with the Milky Way. The velocity of an object projected to the LSR can be written

$$
V_{L S R}=\left[R_{0} \sin (\ell)\left\{\frac{V_{\theta}}{R}-\frac{V_{0}}{R_{0}}\right\}-V_{R} \cos (\ell+\theta)\right] \cos (b)+V_{z} \sin (b),
$$

in an $R, \theta, z$ polar coordinate system centered on the Galactic center, where $\theta$ runs in the direction of Galactic rotation from the Sun-center line, and $z$ is toward the North Galactic Pole. The standard Galactic constants, $R_{0}=8.5 \mathrm{kpc}, V_{0}=220 \mathrm{~km} \mathrm{~s}^{-1}$ are used throughout. If Complex $\mathrm{H}$ moves in a circular orbit $\left(V_{R}=0\right)$ about the Galactic center with a velocity of magnitude $V_{c}$, at $b=0^{\circ}$ it will have components $V_{\theta}=V_{c} \cos (i)$ and $V_{z}=V_{c} \sin (i)$, where $i$ is the inclination of the orbit to the Galactic plane: $i=0^{\circ}$ is prograde in the Galactic plane, and $i=90^{\circ}$ is toward the North Galactic Pole.

The data constrain $V_{z}$ and $V_{\theta}$. At low latitudes $\cos (b) \approx 1$ and $\sin (b) \approx b$, so the gradient $d V_{L S R} / d b \approx V_{z}$. The observed slope in $V_{L S R}$ of the core is about $-3 \mathrm{~km} \mathrm{~s}^{-1}$ per degree of latitude giving $V_{z}=V_{c} \sin (i)=-170 \mathrm{~km} \mathrm{~s}^{-1}$. Constraints on $V_{\theta} / R$ come at $b=0^{\circ}$, where the second term of eq. 1 is zero. At $\ell, b=130^{\circ} .5+0^{\circ}$ the core has $V_{L S R}=-195 \mathrm{~km} \mathrm{~s}^{-1}$ implying that $V_{\theta} / R=-4.3$ $\mathrm{km} \mathrm{s}^{-1} \mathrm{kpc}^{-1}$, or $R=-0.23 V_{c} \cos (i) \mathrm{kpc}$. As the Galactic rotation curve is likely to be nearly flat to several hundred kpc from the Galactic center (Zaritsky 1999), setting $V_{c}=V_{0}=220 \mathrm{~km}$ $\mathrm{s}^{-1}$ yields the orbital inclination $i=230^{\circ}$ and the distance from the Galactic center $R=33 \mathrm{kpc}$. Complex $\mathrm{H}$ thus appears to be moving to greater longitude and to negative latitude at an angle $\approx 45^{\circ}$ with respect to the Galactic plane. The direction of motion is drawn in Fig. 1.

These considerations are used to produce the simulation displayed in Figure 4. Here it is assumed that the Galaxy has a flat rotation curve with $V_{c}=220 \mathrm{~km} \mathrm{~s}^{-1}$ and Complex H has two components, both with $\Delta v=22 \mathrm{~km} \mathrm{~s}^{-1}$. The core follows the retrograde, inclined, orbit $V_{c}=220$ $\mathrm{km} \mathrm{s}^{-1}, i=230^{\circ}$, at a distance $R=33 \mathrm{kpc}$ from the Galactic center. The diffuse gas is slightly more than twice the size of the core and extends to higher latitude; its velocity varies linearly between the velocity of the core where they meet, and normal Galactic disk rotation, $V_{\theta}=V_{0}$ and $V_{z}=0$, at its outer edge. This simulation is not intended to fit the structure of Complex $\mathrm{H}$ in detail, but simply to check the parameters of the proposed model. On this criterion the model does 
well. The simulated core has the correct velocity and gradient, and the lagging diffuse gas produces a credible tail.

Reasonable fits to the GBT data can be found for models in the range $V_{z}=-170 \pm 30 \mathrm{~km} \mathrm{~s}^{-1}$, implying an approximate range of parameters $i=230 \pm 10^{\circ}$ and $R=33 \pm 9 \mathrm{kpc}$. The orbit crosses the Galactic plane at $\theta=38 \pm 5^{\circ}$ from the Sun-center line in the direction of Galactic rotation at a distance from the Sun $r=27 \pm 9 \mathrm{kpc}$. If the Galactic circular velocity declines beyond the Sun as is suggested by some evidence (Zaritsky 1999), a somewhat larger inclination would be derived, along with a smaller value of $R$.

\section{Implied Properties of Complex $\mathbf{H}$}

At a distance from the Sun $r=27 \mathrm{kpc}$ the total H I mass over $V_{L S R} \leq-175 \mathrm{~km} \mathrm{~s}^{-1}$ (the shaded area of Fig. 1) is $6.4 \times 10^{6} M_{\odot}$. About half of this resides in the bright core at $\ell \geq 127^{\circ}, b \leq+5^{\circ}$ while about $10 \%$ is in the diffuse tail at $b>5^{\circ}$. There is certainly $\mathrm{H}$ I associated with the Complex at more positive velocities (e.g., that shown in the Fig. 1 contours), but the restrictions adopted here avoid possible confusion with unrelated material and provide a firm lower limit to the total mass. The size of the core in the $\ell$ and $b$ directions is $\approx 2 \times 3 \mathrm{kpc}$, while the total extent of the cloud is at least $10 \times 5 \mathrm{kpc}$. The dynamical mass needed to bind a spherical cloud of radius $r_{c}$ and line width $\Delta v$ is $M_{d y n} \approx r_{c} \Delta v^{2} / G$, where $G$ is the gravitational constant. The core of Complex $\mathrm{H}$, with $r_{c} \sim 1 \mathrm{kpc}$ and median $\Delta v=22 \mathrm{~km} \mathrm{~s}^{-1}$, has $M_{d y n} \approx 10^{8} M_{\odot}$, while the core H I mass is only $3 \times 10^{6} M_{\odot}$. Complex $\mathrm{H}$ is unlikely to be gravitationally bound by its $\mathrm{H}$ I alone.

The two-phase structure of the $\mathrm{H}$ I lines from the core, also seen in other high-velocity clouds, suggests that it is in pressure equilibrium with a surrounding, low-density, medium (Cram \& Giovanelli 1976; Ferrara \& Field 1994). There is a group of H I features in the Complex at $130^{\circ}+1^{\circ}$ that have a characteristic angular size of $4^{\prime}$ (Wakker \& Schwarz 1991). At $r=27 \mathrm{kpc}$ they would have a diameter of $30 \mathrm{pc}$ and a typical $\langle n\rangle \approx 2 \mathrm{~cm}^{-3}$. Their temperature is likely $50 \mathrm{~K}$ (Wakker, Vijfschaft, \& Schwarz 1991) implying a pressure $P / k \equiv n T \approx 100 \mathrm{~cm}^{-3} \mathrm{~K}$. An identical pressure is derived from the GBT data, using the median core $\Delta v$ of $22 \mathrm{~km} \mathrm{~s}^{-1}$ to limit the temperature, and the median core $N_{H I}$ of $6 \times 10^{19} \mathrm{~cm}^{-2}$ over a size of $2 \mathrm{kpc}$ for an average volume density.

Toward Complex H there is likely $>10^{22} \mathrm{~cm}^{-2}$ of foreground H I. Studies of the region using the 2MASS survey have been complicated by strong differential reddening $(0.6 \lesssim \mathrm{E}(\mathrm{B}-\mathrm{V}) \lesssim 3)$, but no obvious stellar features attributable to Complex $\mathrm{H}$ are found in differenced, dereddened $\left(\mathrm{J}-\mathrm{K}_{s}\right.$, $\left.\mathrm{K}_{s}\right)_{o}$ color-magnitude diagrams compared with nearby control fields (S. Majewski \& M. Skrutskie, private communication). There is an IRAS point source toward Complex $\mathrm{H}$ tentatively identified as a possible young stellar object (Ivezić \& Christodoulou 1997), and weak $H_{\alpha}$ emission toward the brightest part of the Complex (Putman et al, in press), but the $H_{\alpha}$ velocity is $30 \mathrm{~km} \mathrm{~s}^{-1}$ more positive than the velocity of the core and does not correspond to any feature in $\mathrm{H} \mathrm{I}$. 


\section{Summary Comments}

The model derived from the kinematics of Complex $\mathrm{H}$ is consistent with the overall structure of the object. The large-scale "head-tail" morphology seen in Fig. 1 (the trend continues to even more positive velocities than are shown) is the form one would expect from a cloud containing dense concentrations moving on an inclined, retrograde orbit to higher $\ell$ and lower $b$, shedding gas through interaction with the Milky Way. A number of high-velocity clouds believed to be interacting with the Milky Way also have this structure, where, like Complex H, the velocity of the diffuse gas "downstream" in the tail is closer to Galactic rotation than the gas in the head (Pietz et al 1996; Brüns et al. 2000). The physical issues involved in the interaction of a high-velocity cloud with an external medium have stimulated considerable theoretical interest (Benjamin 1999); magnetic fields may be important in preserving the integrity of the main cloud (Santillan et al 1999; Konz, Brüns, \& Birk 2002; Quilis \& Moore 2001).

In its small-scale structure Complex H resembles parts of the Magellanic Stream. Though its origin is tidal, the Stream shows evidence for interaction with an external medium (Putman et al 1998; Konz, Brüns, \& Birk 2002). It contains H I in two phases, and clouds with a size of 15-30 pc, $\langle n\rangle \approx 2-6 \mathrm{~cm}^{-3}$, and $\Delta v \approx 5 \mathrm{~km} \mathrm{~s}^{-1}$, virtually identical to the knots in Complex $\mathrm{H}$ (Wakker, Oosterloo, \& Putman 2002). In its large-scale characteristics, Complex H resembles the dwarf irregular galaxy Leo A. At a distance of $0.8 \mathrm{Mpc}$ it has about the same $\mathrm{H}$ I mass as the Complex and the same linear size as the core; the $\mathrm{H}$ I lines from the two objects have a similar width and two-component structure (Young \& Lo 1996; Schulte-Ladbeck et al 2002). Most dwarf irregular galaxies, however, have considerably more H I mass than Complex H (Mateo 1998). The Complex resembles as well some "compact" high-velocity H I clouds which, if they are at distances of several hundred kpc, have a similar size and $\mathrm{H}$ I mass and also have two H I phases (Burton, Braun, \& Chengalur 2001; de Heij, Braun, \& Burton 2002).

Complex H may be interacting with the Galactic halo or some part of the disk. Its orbital period for $V_{c}=220 \mathrm{~km} \mathrm{~s}^{-1}$ is $\sim 10^{9} \mathrm{yr}$ over which time it traverses heights of $\pm 25 \mathrm{kpc}$ from the Galactic plane. There is convincing evidence for a gaseous halo that extends to at least $R \sim 50$ kpc toward the Magellanic Stream, though little is known of it in detail (Savage 1995; Kalberla \& Kerp 1998; Konz, Brüns, \& Birk 2002; Savage et al 2003). The normal H I disk emission at $\ell \approx 130^{\circ}$ goes to $V_{L S R} \approx-110 \mathrm{~km} \mathrm{~s}^{-1}$ which, for a flat rotation curve, is $R=25 \mathrm{kpc}$, similar to the derived distance of the Complex. The Galaxy may also have an extended disk of ionized gas like some other systems (Bland-Hawthorn, Freeman, \& Quinn 1997). The existing GBT H I data do not cover enough area to be useful in evaluating the suggestion of Morras, Bajaja, \& Arnal (1998) that Complex $\mathrm{H}$ has actually pierced the $\mathrm{H}$ I disk, but further observations are planned.

Galaxies exist in a rich environment of companions, interaction and distortion (Sancisi 1999). Retrograde motions are observed in H I components of many systems (Bertola \& Corsini 1998), and in Milky Way globular clusters (Freeman \& Bland-Hawthorn 2002). A retrograde orbit at large R tends to be less destructive to a satellite galaxy than a prograde orbit (Toomre \& Toomre 1972; 
Hernquist \& Quinn 1988), which may account for the persistence of the object. But Complex $\mathrm{H}$ may not persist much longer. If a complete accounting of the mass of diffuse, presumably decelerated, gas associated with the Complex could be made, it might well show that the coherent core is only a fraction of the total $\mathrm{H} \mathrm{I}$, implying that Complex $\mathrm{H}$ is now in the process of being destroyed.

Finally, the guiding assumption that Complex $\mathrm{H}$ is in a purely circular orbit (or is just now at perigalacticon) is clearly too naive - there is likely some ellipticity and a radial velocity component. Additional data will supply extra constraints on $V_{\theta}$. But the simple model presented here, motivated by the new evidence for interaction with the Galaxy, suggests that the velocity of Complex $\mathrm{H}$ and its gradient with latitude carry significant information about its space motion, and are not entirely contingent.

I thank R.A. Benjamin, W.B. Burton, S. Majewski, E.M. Murphy, T.M. Tripp, and the referee, H. van Woerden, for useful discussions and comments on the manuscript.

\section{REFERENCES}

Benjamin, R.A. 1999, in Stromlo Workshop on High Velocity Clouds, ASP Conf. Ser. Vol. 166, p. 147

Bertola, F., \& Corsini, E. 1998, in Galaxy Interactions at Low and High Redshift, IAU Symp. 186, ed. J.E. Barnes \& D.B. Sanders, Kluwer, p. 149

Bland-Hawthorn, J., Freeman, K.C., \& Quinn, P.J. 1997, ApJ, 490, 143

Blitz, L., Spergel, D.N., Teuben, P., Hartmann, D., \& Burton, W.B. 1999, ApJ, 514, 818

Brüns, C., Kerp, J., Kalberla, P.M.W., \& Mebold, U. 2000, A\&A, 357, 120

Burton, W.B., Braun, R., \& Chengalur, J.N. 2001, A\&A, 375, 227

Cram, T.R., \& Giovanelli, R. 1976, A\&A, 48, 39

Dieter, N.H. 1971, A\&A, 12, 59

Ferrara, A., \& Field, G.B. 1994, ApJ, 423, 665

Freeman, K., \& Bland-Hawthorn, J. 2002, ARA\&A, 40, 487

Hartmann, D. \& Burton, W.B., 1997, "Atlas of Galactic Neutral Hydrogen”, Cambridge University Press

de Heij, V., Braun, R., \& Burton, W.B. 2002, A\&A, 391, 67 
Hernquist, L. \& Quinn, P.J. 1988, ApJ, 331, 682

Hulsbosch, A.N.M. 1971, A\&A, 14, 489

Hulsbosch, A.N.M. 1975, A\&A, 40, 1

Ivezić, Ž., \& Christodoulou, D.M. 1997, ApJ, 486, 818

Kalberla, P.M.W., \& Kerp, J. 1998, A\&A, 339, 745

Konz, C., Brüns, C., \& Birk, G.T. 2002, A\&A, 391, 713

Lockman, F.J. 2002, ApJ, 580, L47

Mateo, M. 1998, ARA\&A, 462, 203

Morras, R., Bajaja, E., \& Arnal, E.M. 1998, A\&A, 334, 659

Pietz, J., Kerp, J., Kalberla, P.M.W., Mebold, U., Burton, W.B. \& Hartmann, D. 1996, A\&A, 308, L37

Putman, M.E. et al 1998, Nature, 394, 752

Quilis, V., \& Moore, B. 2001, ApJ, 555, L95

Sancisi, R. 1999, in Galaxy Interactions at Low and High Redshift, IAU Symposium 186, ed. J.E. Barnes \& D.B. Sanders, Kluwer, p. 71

Santillan, A., Franco, J., Martos, M., \& Kim, J. 1999, ApJ, 515, 657.

Savage, B.D. 1995, in The Physics of the Interstellar Medium and Intergalactic Medium, ed. A. Ferrara, C.F. McKee, C. Heiles, \& P.R. Shapiro, ASP Conf. Ser. 80, p. 233

Savage, B.D., Sembach, K.R., Wakker, B.P., Richter, P., Meade, M., Jenkins, E.B., Shull, J.M., Moos, H.W., \& Sonneborn, G. 2003, ApJS, 146, 125

Schulte-Ladbeck, R.E., Hopp, U., Drozdovsky, I.O., Greggio, L., \& Crone, M.M. 2002, AJ, 124, 896

Toomre, A., \& Toomre, J. 1972, ApJ, 178, 623

Wakker, B.P., Vijfschaft, B., \& Schwarz, U.J. 1991, A\&A, 249, 233

Wakker, B.P., \& Schwarz, U.J. 1991, A\&A, 250, 484

Wakker, B.P., van Woerden, H., de Boer, K., \& Kalberla, P. 1998, ApJ, 493, 762.

Wakker, B.P., Oosterloo, T.A., \& Putman, M.E. 2002, AJ, 123, 1953

Young, L. \& Lo, K.Y. 1996, ApJ, 462, 203 
Zaritsky, D. 1999, in The Third Stromlo Symposium: The Galactic Halo, ed. B.K. Gibson, T.S. Axelrod, M.E. Putman, ASP Conf. Ser. Vol. 165, 34 


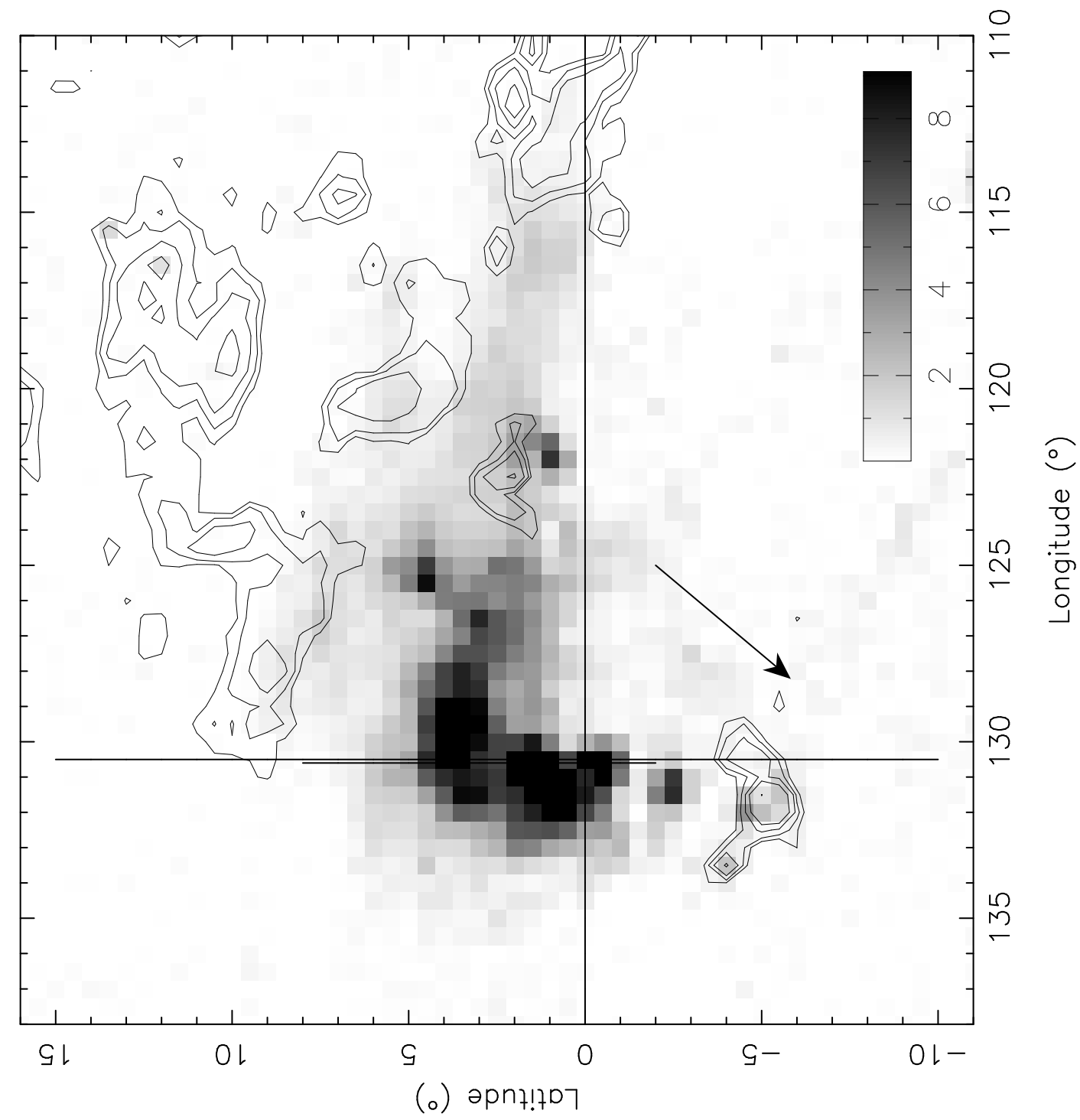

Fig. 1.- Complex H in the Leiden-Dwingeloo 21cm H I survey data (Hartmann \& Burton 1997) at $35^{\prime}$ angular resolution. The shading shows $\mathrm{H}$ I at $V_{L S R} \leq-175 \mathrm{~km} \mathrm{~s}^{-1}$, which includes the brightest $\mathrm{H}$ I in the Complex. The contours trace the $\mathrm{H}$ I emission at more positive velocities between -175 and $-140 \mathrm{~km} \mathrm{~s}^{-1}$, closer to that allowed by normal Galactic rotation. The vertical lines near $\ell=130^{\circ}$ show the locations of the velocity-latitude cuts of Figs. 2 and 3, which were used to derive the model for the cloud's motion in the direction shown by the arrow. In this model, most of the contoured material would have been decelerated by interaction with the Milky Way. The grey scale is in units of $10^{19} \mathrm{~cm}^{-2}$; coutours are equally spaced every $2 \times 10^{19} \mathrm{~cm}^{-2}$. 


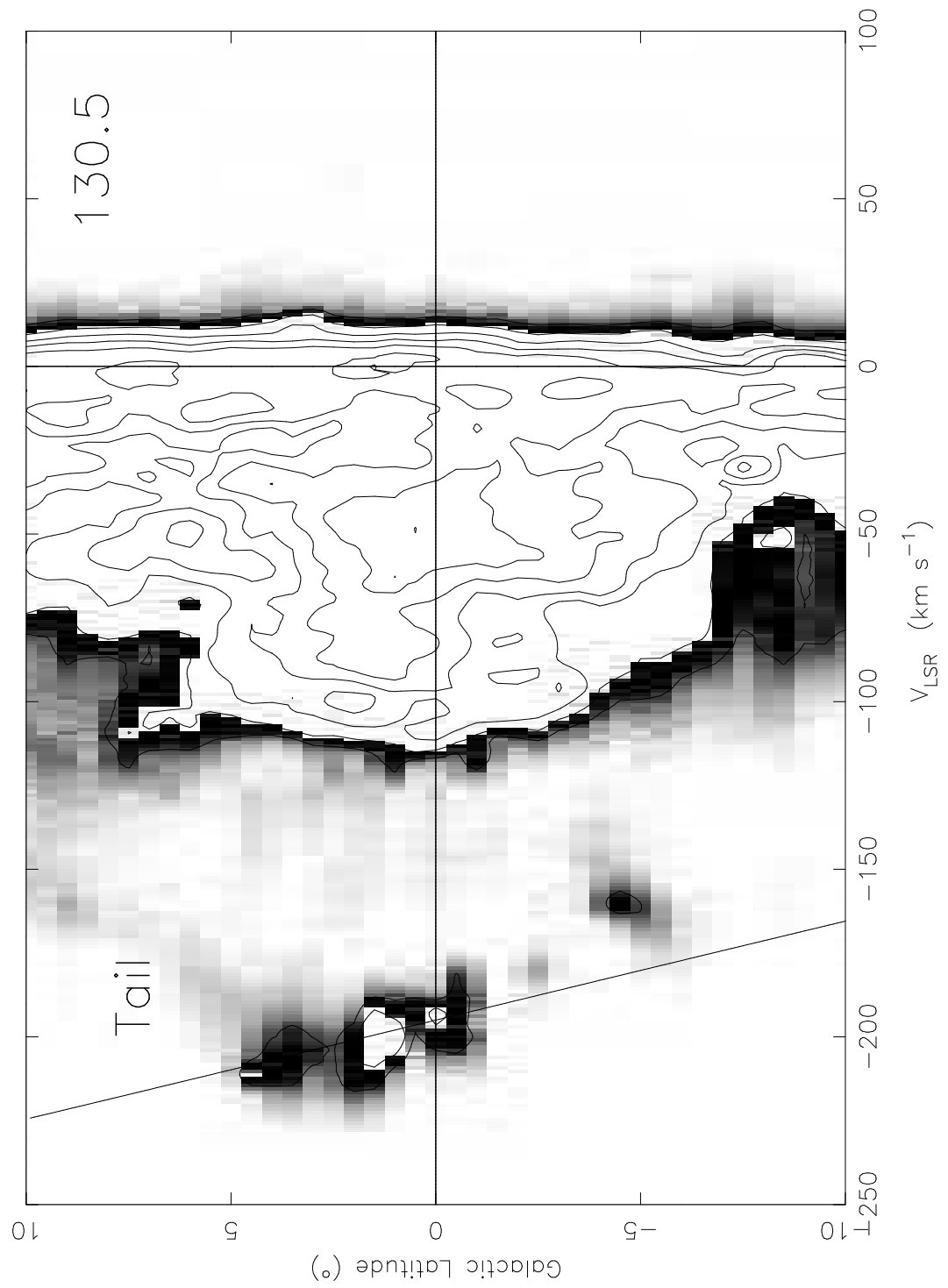

Fig. 2.- A velocity-latitude cut (a "slit spectrum") through Complex $\mathrm{H}$ at $\ell=130.5$ from the LD survey data showing the "core" at $b<5^{\circ}$ and the "tail" at $b>5^{\circ}$. The slanting line through the core follows $V_{L S R}=-195-170 \sin (b) \mathrm{km} \mathrm{s}^{-1}$. The slope of the line gives the vertical component of orbital motion, $V_{z}$, while its value at $b=0^{\circ}$ constrains $V_{\theta} / R$. Contours are in factors of two from the peak; the gray scale shows emission at $T_{b} \leq 3 \mathrm{~K}$. 

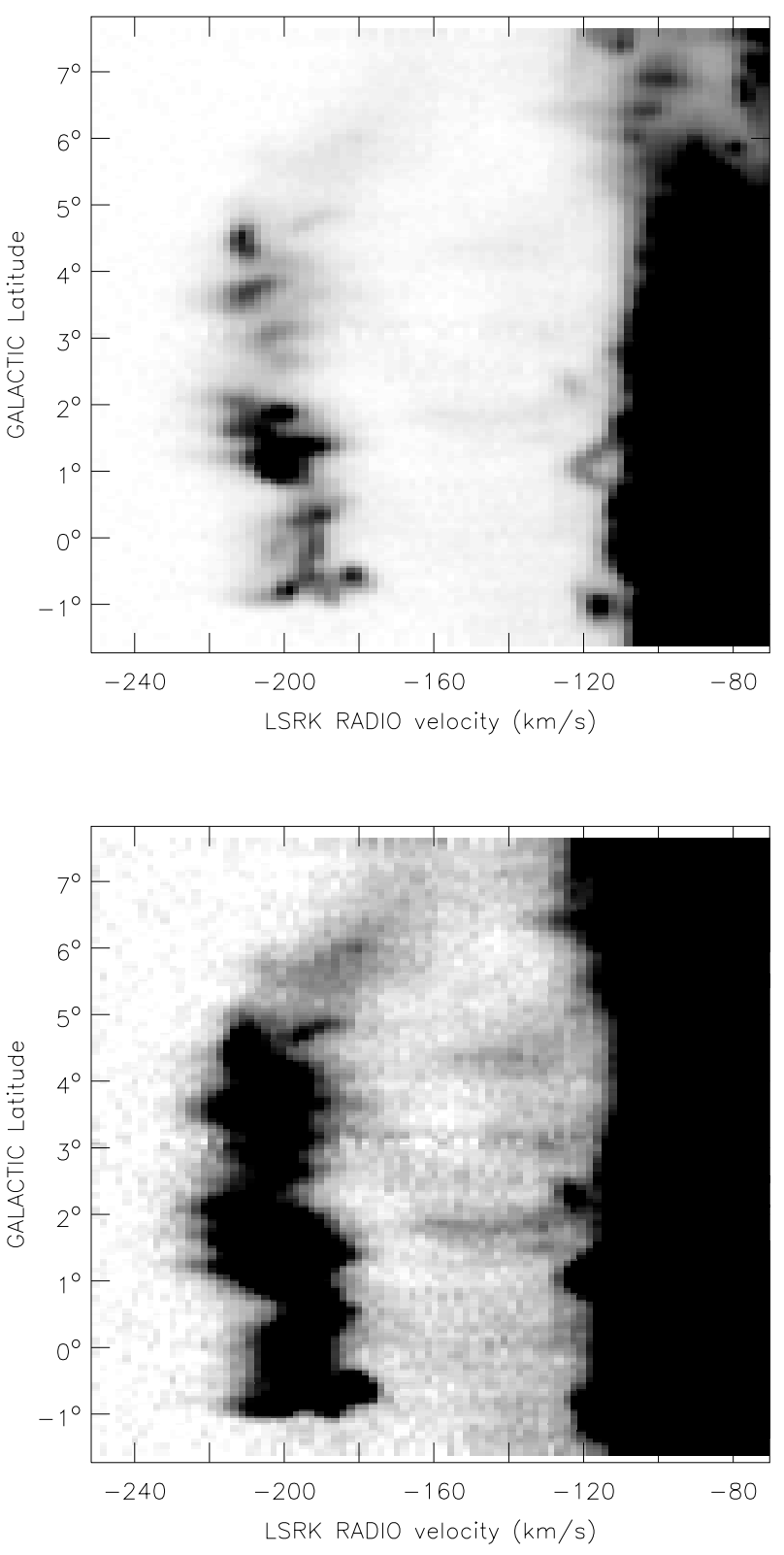

Fig. 3. - A velocity-latitude cut through Complex $\mathrm{H}$ at $\ell=130.6$ made from GBT data at $9^{\prime}$ angular resolution. The two panels show identical data; the upper panel is clipped at $5 \mathrm{~K}$, the lower at $1 \mathrm{~K}$. The "tail" at $b>5^{\circ}$ is almost entirely broad-line gas while the bright "core" at $b<5^{\circ}$ contains many knots of narrower lines. There is diffuse $\mathrm{H}$ I emission at velocities between Complex $\mathrm{H}$ and the Galactic disk suggesting that some material from the Complex has been decelerated by interaction with the Milky Way. 


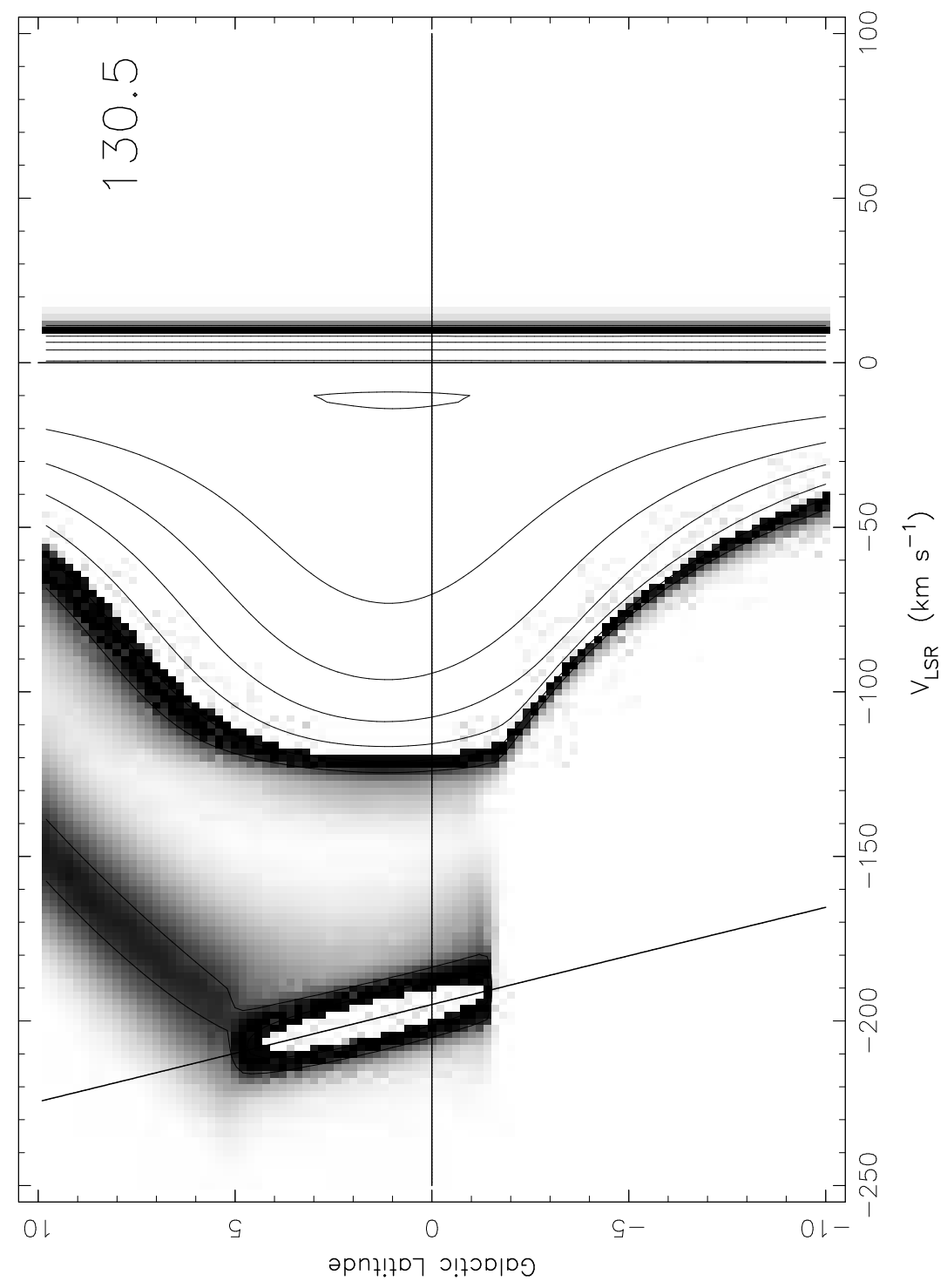

Fig. 4.- Simulation of Complex $\mathrm{H}$ as a cloud in an inclined retrograde orbit surrounded by a diffuse component whose velocity changes linearly between the retrograde core and normal prograde Galactic rotation. The simulation is intended to match the observations of Figs. 2 and 3. The velocity tilt of the core results from the projection of its vertical motion. The diagonal line is the same one drawn through the data in Figure 2. 